\title{
Neurally Adjusted Ventilatory Assist Mode in Pediatric Intensive Care Unit and Pediatric Cardiac Care Unit
}

\author{
Monika Gupta ${ }^{1,2,3^{*}}$, Maria Bergel ${ }^{1,3}$, Nicole Betancourt ${ }^{1,3}$ and Vicki L. Mahan ${ }^{1,2,4}$ \\ ${ }^{1}$ St Christopher's Hospital for Children, Philadelphia, PA, USA; ${ }^{2}$ Drexel University College of Medicine, Philadelphia, PA, USA; ${ }^{3}$ Section \\ of Critical Care Medicine, Department of Pediatrics, St Christopher's Hospital for Children, Philadelphia, PA, USA; ${ }^{4}$ Section of Pediatric \\ Cardiothoracic Surgery, Department of Pediatric Surgery, St Christopher's Hospital for Children, Philadelphia, PA, USA
}

\begin{abstract}
Neonatal, cardiac and pediatric intensive care units (ICUs) frequently use the neurally adjusted ventilatory assist (NAVA) mode of ventilation, which has been shown to improve patient-ventilator synchrony and decrease work of breathing as it uses neural impulse to guide patient and ventilator breaths. We reviewed uses of NAVA as the mode of ventilation and oxygenation in pediatric (P)ICU and cardiac (C)ICU patients. We found that NAVA mode improves patient-ventilator interaction by improving synchrony and thus decreasing work of breathing. This eventually improved all aspects of critical care, as demonstrated by decreased need for sedation, improvement in hemodynamics, improvement in patient comfort, decreased days on ventilator, decreased days in ICU and decreased hospital length of stay. Additional studies need to be done in order to help NAVA technology become a primary mode of ventilation in PICU and CICU. Further studies are also needed to evaluate the successful use of noninvasive-NAVA as a means of preventing endotracheal intubation or to allow early extubation in our population.
\end{abstract}

\section{Introduction}

Conventional modes of ventilation currently used in pediatric and cardiac intensive care units (PICUs and CICUs, respectively) include volume, pressure or combination modes. These are mostly assist modes, as they are delivered by means of pneumatic signals generated by patient effort. Though these currently serve the purpose of oxygenation and ventilation, these are not ideal due to asynchrony and effect on patient's work of breathing.

Neurally adjusted ventilator assist (NAVA) mode was predominantly developed by Sinderby et al. to promote comfort in neo-

Keywords: Neural; Mechanical ventilation; Respiratory failure.

Abbreviations: NAVA, neurally adjusted ventilatory assist; NIV-NAVA, non invasive neurally adjusted ventilatory assist; PICU, pediatric intensive care unit; CICU, cardiac intensive care unit; Edi, electrical activity of diaphragm; Edi-min, electrical activity of diaphragm minimum; NEX, nose- ear- xiphoid; PEEP, Positive End-Expiratory Pressure; PIP, peak inspiratory pressure; EKG, electro-cardio-gram; $\mu \mathrm{V}$, microvolts; PC, pressure control; PS, pressure support; PRVC, pressure regulated volume control; RSV, respiratory syncytial virus; COMFORT, comfort scale; CDH, congenital diaphragmatic hernia; ARDS, acute respiratory distress syndrome; SIMV, synchronized intermittent mechanical ventilation; nCPAP, nasal continuous positive airway pressure; NIPPV, nasal intermittent positive pressure ventilation.

Received: December 19, 2016; Revised: March 23, 2017; Accepted: April 18, 2017

*Correspondence to: Monika Gupta, Drexel University College of Medicine and Dentistry, 160 East Erie Ave, Philadelphia, PA 19134-1095, USA. Tel: (215) 427 8812, Fax: (215) 427 5525, E-mail: monika.Gupta@tenethealth.com; monika_met@ yahoo.com

How to cite this article: Gupta M, Bergel M, Betancourt N, Mahan VL. Neurally Adjusted Ventilatory Assist Mode in Pediatric Intensive Care Unit and Pediatric Cardiac Care Unit. Exploratory Research and Hypothesis in Medicine 2017;2(2):33-37. doi: 10.14218/ERHM.2016.00027 nates. ${ }^{1-3}$ To understand this novel mode of ventilation, we must first understand the physiology of normal ventilation. A variety of signals constantly provide neural feedback via complex regulatory systems, such as stretch receptors in the lungs, the HeringBreuer reflex, lung compliance changes, upper airway receptors, peripheral and central chemoreceptors. These signals originate in the respiratory center of the brain stem and are then carried via the phrenic nerve to the diaphragm resulting in diaphragmatic contraction and subsequent chest expansion. This process generates negative pressure in the thoracic cavity, drawing in air and causing expansion of the lungs and consequent changes in pulmonary pressure, flow and volume. ${ }^{1-4}$

NAVA utilizes electrical voltages travelling via the phrenic nerve (known as electrical activity of the diaphragm (Edi); Fig. 1) as a marker of respiratory effort in guiding the ventilator for initiation of respirations. A specialized nasogastric tube, with eight bipolar electrodes (sensors), is positioned in the lower esophagus at the level of the crural diaphragm to measure the Edi signal (Figs. 2-4).

As seen in Figure 1, in a healthy volunteer in the resting state, low Edi voltages can maximally recruit diaphragmatic muscles, generating a normal tidal volume (green diaphragm). However, when patients initially become sick (yellow diaphragm), higher Edi voltages are needed to achieve the same tidal volume. Sicker patients (red diaphragm) maximize Edi voltages from the neural center and are unable to generate adequate tidal volumes.

\section{Edi signals}

The Edi signal depends on nerve fiber recruitment (i.e. the number 


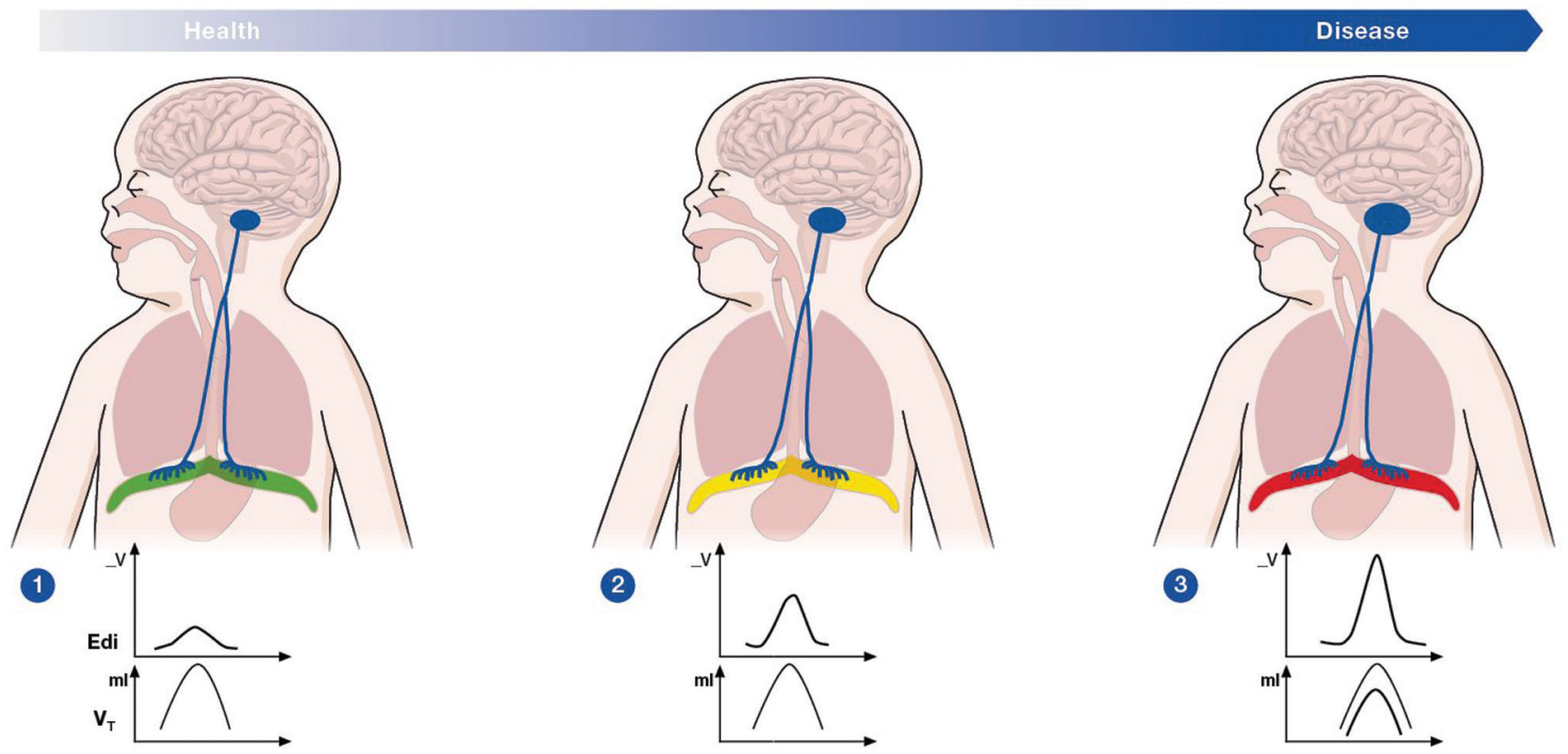

Fig. 1. Edi signal in healthy vs. sick patients. Abbreviation: Edi, Electrical activity of diaphragm.

of neurons that are sending the stimulus) and patient's respiratory rate. Signals from each electrode pair are differentially amplified, digitized and processed by specialized NAVA software. Electrical contamination from the heart, esophagus and environment are filtered and silenced to obtain the highest possible signal-to-noise ratio. In patients on NAVA, the following anatomic factors do not influence Edi signals: lung volume, body position, intra-abdominal pressure, postural and expiratory muscles, subcutaneous layers, applied positive end-expiratory pressure (PEEP), nasogastric feeds or milk influx during oral feeding.

Factors that decrease or alter Edi signals include failure to deliver respiratory signals due to apnea of prematurity, central hypoventilation syndrome, over-assist, hyperventilation, brain injury and sedation, as well as anatomic reasons (i.e. diaphragmatic hernia) and peripheral abnormalities such as phrenic nerve conduction failure, disease, or chemical paralysis of the neuromuscular junction or diaphragm.

\section{Placement of the Edi catheter}

Edi catheter placement, similar to nasogastric or oro-gastric tubes, uses measurements from nose to ear to xiphoid distance. The formula for insertion is included in the insert of the package from the manufacturer of the representative catheter shown in Figure 2 (Maquet, Rastatt, Germany).

\begin{tabular}{|ll|}
\hline Insertion distance $Y$ for nasal insertion \\
\hline r $/ \mathrm{cm}$ & Calculation of $Y$ \\
$8 \mathrm{Fr} 100 \mathrm{~cm}$ & NEX cm $\times 0.9+8=Y \mathrm{~cm}$ \\
$6 \mathrm{Fr} 50 \mathrm{~cm}$ & NEX cm $\times 0.9+3.5=Y \mathrm{~cm}$ \\
$6 \mathrm{Fr} 49 \mathrm{~cm}$ & NEX cm $\times 0.9+2.5=Y \mathrm{~cm}$ \\
\hline
\end{tabular}

Fig. 2. Insertion distance for nasogastric Edi Catheter. Abbreviations: Edi, Electrical activity of diaphragm; NEX, Nose- Ear- Xiphoid.
As seen in Figure 5, correct catheter position is demonstrated by the presence of the largest $p$-waves in the upper leads and by QRS complexes in the middle leads. There is subsequent progression to minimal or absent $\mathrm{p}$-waves and QRS complexes in the lower leads. The Edi signal is superimposed on the retro-cardiac electrocardiogram as a lavender/purple color on the second and third lead.

The highest Edi value of the waveform (Edi peak) represents neural inspiratory effort and is responsible for the size and duration of the breath. The lowest Edi (Edi min) represents spontaneous tonic activity of the diaphragm, which prevents de-recruitment of alveoli during expiration. The Edi trigger $(\mu \mathrm{V}$; the minimum increase in electrical activity from the previous trough) triggers the

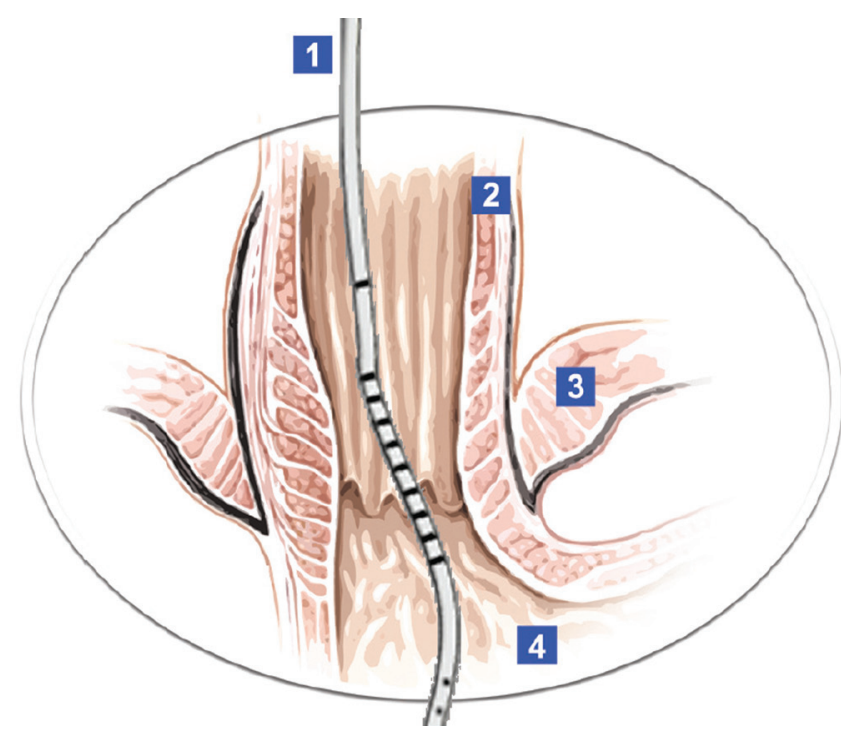

Fig. 3. Edi catheter in relation to diaphragm. 1. Edi catheter; 2. Esophagus; 3. Diaphragm; 4. Gastric mucosa. Abbreviation: Edi, Electrical activity of diaphragm. 


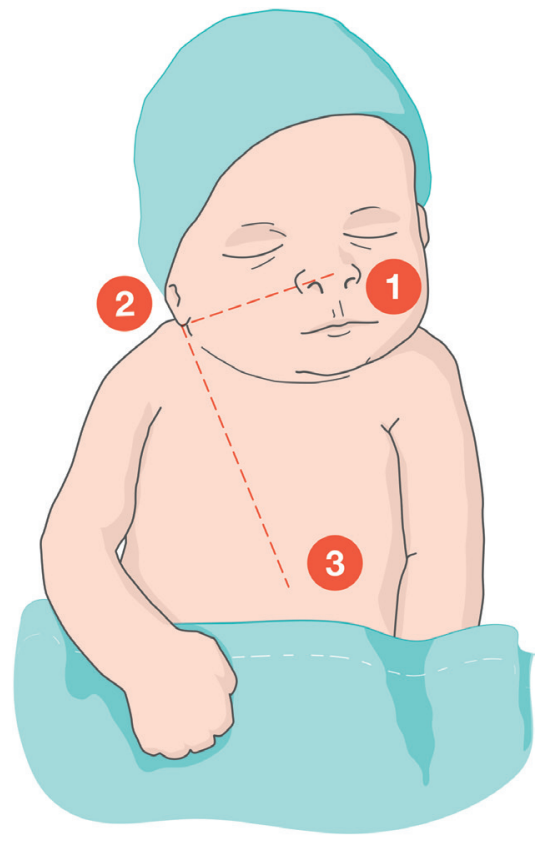

Fig. 4. Measurement for Edi catheter placement. 1: Nose, 2: Ear, 3: Xiphoid process. Abbreviation: Edi, Electrical activity of diaphragm.

ventilator to recognize the increase in electrical activity, thereby resulting in initiation of a breath.

The NAVA level $\left(\mathrm{cmH}_{2} \mathrm{O} / \mu \mathrm{V}\right.$; a conversion factor) translates the Edi signal into proportional pressures. Edi is multiplied by the NAVA level to determine airway pressure delivered by the ventilator for each breath. The NAVA software automatically calculates peak inspiratory pressure (PIP) every 17 minutes. This is demonstrated by the formula

$$
\text { PIP }=\text { NAVA level } \times(\text { Edi peak }- \text { Edi min })+\text { PEEP. }
$$

\section{Setting up NAVA as mode of ventilation}

Setting up NAVA mode of ventilation involves the set-up of NAVA, NAVA pressure support (PS) mode and NAVA backup pressure control (PC) mode, enabling the patient to transition between all three modes. The ventilator has a preset apnea time default; however, this can be altered based on the patient's age and respiratory rate. This ensures safety of patients should the Edi catheter disconnect or upon leakage of signals, absence of patient respiratory effort or other reasons for asynchrony. Clinical comfort and blood gases are used to determine the appropriate level of support. Edi peak may be influenced by the amount of ventilatory support provided. Overventilation can suppress spontaneous respiratory drive and decrease the Edi signal. Underventilation can result in increased respiratory drive and higher Edi signals. ${ }^{1-4}$

\section{Safety mechanisms for NAVA}

In order to safely promote intermittent lung recruitment, the patient's ability to generate sufficient inspiratory pressure should be considered when setting a PIP limit. The PIP limit is set to prevent excessively large breaths; the ventilator will waste excessive tidal volume at $5 \mathrm{cmH}_{2} \mathrm{O}$ below the set PIP limit. A level that is set too low results in an inability to generate a sigh or recruiting breaths; this may result in the development of progressive atelectasis.

Should the Edi catheter become mal-positioned, dislodged, or fail to detect the Edi signal, the ventilator reverts to NAVA PS. In a clinically apneic patient, the ventilator switches to NAVA PC. Upon Edi signal return, NAVA reverts to previous settings.

The ventilator delivers inspiratory pressure only as long as the Edi signal is detected. At the point of optimal lung inflation, the Hering-Breuer reflex is stimulated. This stretch reflex functions as a negative feedback mechanism to terminate the Edi signal and arrest ventilator flow, protecting the lung from overinflation.

\section{Use of NAVA mode of ventilation in PICU and CICU patients}

Optimization of assist-ventilation with NAVA, without the delays seen in conventional ventilation, has been proven over multiple cross-over studies in the pediatric and infant population, as well as in at least one parallel pilot study. ${ }^{5}$ Invariably, these studies have shown a decrease in asynchrony in the infant and pediatric populations when compared to both PC and PS conventional ventilation. Similar results were shown in a 10-patient infant study by Bordessoule et al. in $2012 .^{6}$ The authors demonstrated statistically significant decreases in trigger delay, asynchrony index, wasted effort and an increased correlation between the ventilator and Edi. A separate study demonstrated an asynchrony index of $0 \%$ in 14 preterm neonates over the course of 12 hours of NAVA ventilation as compared to $22 \%$ in neonates ventilated in pressure regulated volume control (PRVC) mode. ${ }^{7}$ Investigators have also demonstrated a total of 1,841 neural apneas during PRVC ventilation, with a median duration of 29 seconds; patients receiving NAVA ventilation had no neural apneas. This was hypothesized to be related to overventilation and support while in the PRVC mode. ${ }^{7}$ In another study by Liet et al., the use of NAVA in children admitted with respiratory distress secondary to respiratory syncytial virus bronchiolitis was found to be effective because of its ability to minimize asynchrony. ${ }^{8}$ Based on findings of improved oxygenation and lower inspiratory pressures, the authors hypothesized that synchrony improves assist delivery, prevents air swallowing and gastric distention, and improves pulmonary gas exchange, systemic blood flow and oxygen supply to tissues, decreasing morbidity, duration of ventilation and hospital length of stay. ${ }^{8}$ Additionally, Piastra and colleagues showed significant decreases in PIP, overall decrease in mean airway pressure and heart rate. ${ }^{5}$ The authors also demonstrated improved COMFORT score in patients under 1 year of age who were weaned from high frequency oscillatory ventilation to extubation via NAVA, as opposed to pressure supported ventilation. ${ }^{5}$

In patients with acute respiratory distress syndrome (ARDS), NAVA could potentially be associated with significant physiological advantages over conventional ventilation. ARDS results in variable breathing patterns that affect metabolic demands, acid-base status and respiratory system mechanical properties. The preliminary physiological findings obtained by a small study conducted by Terzi et al. suggest that neural triggering provides a more effective means of weaning mechanical ventilation in patients with ARDS, while minimizing the hazards of dynamic hyperinflation. ${ }^{9}$ The aforementioned 10-patient study of infants $<1$ year of age also demonstrated improved breath-to-breath variability with NAVA, providing corresponding variable pressure delivery in comparison to PC and PS ventilation. ${ }^{6}$

The successful use of NAVA in a patient with congenital diaphragmatic hernia $(\mathrm{CDH})$ pre- and post-repair has also been de- 


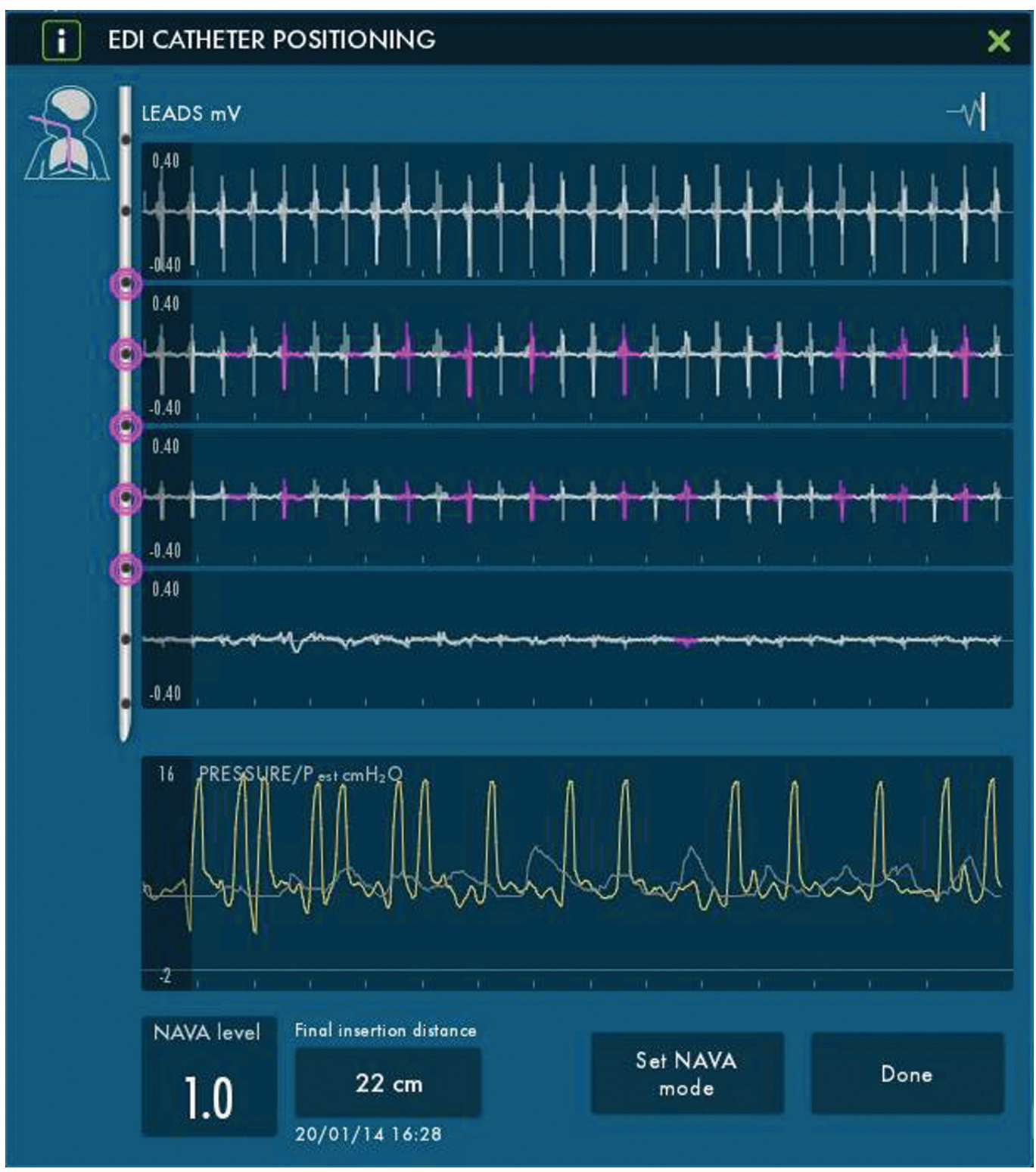

Fig. 5. Display on ventilator confirming appropriate placement of Edi catheter. Abbreviation: Edi, Electrical activity of diaphragm.

scribed in a single case report. ${ }^{10}$ In this case, NAVA was implemented after synchronized intermittent mechanical ventilation PC/ PS failed within 24 hours of intubation secondary to asynchrony and discomfort. Upon switching to NAVA, the patient appeared more comfortable, resulting in sedation wean. Minehart et al. demonstrated that despite a defect in the diaphragm, Edi signals allowed for synchronized ventilation pre- and post-operatively. The child was extubated on postoperative day $1 .{ }^{10}$ Of note, there are additional case reports on the use of NAVA in patients post-CDH repair.

Data is very limited regarding use of NAVA in postoperative congenital cardiac patients. In 21 postoperative cardiac patients, Bengtsson et al. demonstrated safe and potentially efficacious use of NAVA, improvement in patient-ventilator synchrony, reduction in airway pressures and trends towards early extubation. ${ }^{11}$ In another study, Zhu and colleagues compared hemodynamic safety, oxygenation and gas exchange effects on patients with NAVA and pressure ventilation. They confirmed that there is no effect on hemodynamics in most of the postoperative cardiac patients. ${ }^{12}$ Additionally, they found that the P/F ratio in the NAVA group was slightly higher than conventionally ventilated patients. ${ }^{12}$ More studies are needed in this area to help bring this technology into mainstream practice.

Future research directions

The majority of literature on the clinical use of NAVA is based on its use in intubated patients. However, there are studies that demonstrate the successful use of noninvasive NAVA (NIV-NAVA) in neonates. Firestone et al. evaluated PIPs and Edi in premature infants using NIV-NAVA respiratory support. ${ }^{13}$ In a study by Longhini et al. comparing the use of NAVA before and after extubation, there was no difference found in patient-ventilator interaction, gas 
exchange, sedation requirements or vital signs between invasive and NIV-NAVA. ${ }^{14}$ There is, however, a trend in pediatric critical care towards the use of nasal continuous positive airway pressure or nasal intermittent positive pressure ventilation in order to potentially prevent intubation and to support the patient's cardiorespiratory status post-extubation. Therefore, there is a probable role for the use of NIV ventilation with NAVA, particularly in neonates and infants in our PICU.

As we have seen in our PICU, the infants receiving NIV ventilation for acute respiratory failure often fail to synchronize with the ventilator, resulting in inefficient ventilation and oxygenation. Asynchrony during ventilation can have numerous adverse effects on the infant, namely increased mean airway pressure, fraction of inspired oxygen requirements, risk of barotrauma, risk and complications of sedation and risk of hemodynamic instability. ${ }^{13}$ The use of NIV-NAVA in our neonate and infant populations could provide more effective synchronized ventilation that is more comfortable and less harmful and could also potentially lead to successful early extubation and decrease need for re-intubation. Additional studies need to be done in order to evaluate the successful use of NIVNAVA as a means of preventing endotracheal intubation or to allow early extubation in our population.

\section{Limitations}

While NAVA improves arterial oxygenation and alveolar ventilation in pediatric patients by way of comfortable, synchronous ventilation with lower peak airway pressures, there are some significant drawbacks to this technology. NAVA is a new mode of ventilation, requiring some degree of adaptation and education in order to achieve the aforementioned benefits. Also, the Edi catheter is relatively expensive, although it does have the additional capability of use for feeding. ${ }^{9}$ The NAVA technology itself is also expensive, due to the need for specialized software on the ventilators and frequent changing of the Edi catheters.

\section{Conclusions}

NAVA ventilation is an innovative way of providing more effective ventilation to intubated patients in the critical care setting using a sensitive physiologic trigger to initiate breaths. The comparative NAVA studies have demonstrated improved patientventilator synchrony with adequate oxygenation and ventilation. As the field of critical care (PICU and CICU) continues to favor less invasive interventions, there is much potential to use NIVNAVA in order to prevent reintubation and facilitate successful early extubation.

\section{Conflict of interest}

The authors have no conflict of interests related to this publication.
Author contributions

Literature search (MG, MB, NB, VLM), writing sections of the manuscript and supervision (MG, VLM), writing pediatric cardiac care section of the manuscript (MB), writing pediatric intensive care section of the manuscript (NB).

\section{References}

[1] Maquet. Pocket Guide: NAVA ${ }^{\circledR}$ and NIV NAVA in neonatal settings. 2010.

[2] Courtesy of Stein H, Firestone K, Synderby C, Beck J. Materials from Workshop on NAVA. Toledo Ohio, Sept 2014.

[3] Stein H, Firestone K. Application of neurally adjusted ventilatory assist in neonates. Semin Fetal Neonatal Med 2014;19(1):60-69. doi:10.1016/j.siny.2013.09.005.

[4] Kacmarek RM. Proportional assist ventilation and neurally adjusted ventilatory assist. Respir Care 2011;56(2):140-148. doi:10.4187/ respcare.01021.

[5] Piastra M, De Luca D, Costa R, Pizza A, De Sanctis R, Marzano L, et al. Neurally adjusted ventilatory assist $v s$ pressure support ventilation in infants recovering from severe acute respiratory distress syndrome: nested study. J Crit Care 2014;29(2):312.e1-312.e5. doi:10.1016/j. jcrc.2013.08.006.

[6] Bordessoule A, Emeriaud G, Morneau S, Jouvet P, Beck J. Neurally adjusted ventilatory assist improves patient-ventilator interaction in infants as compared with conventional ventilation. Pediatr Res 2012;72(2):194-202. doi:10.1038/pr.2012.64.

[7] Longhini F, Ferrero F, De Luca D, Cosi G, Alemani M, Colombo D, et al. Neurally adjusted ventilatory assist in preterm neonates with acute respiratory failure. Neonatology 2015;107(1):60-67. doi:10.1159/ 000367886.

[8] Liet JM, Dejode JM, Joram N, Gaillard-Le Roux B, Bétrémieux P, Rozé JC. Respiratory support by neurally adjusted ventilatory assist (NAVA) in severe RSV-related bronchiolitis: a case series report. BMC Pediatr 2011;11:92. doi:10.1186/1471-2431-11-92.

[9] Terzi N, Pelieu I, Guittet L, Ramakers M, Seguin A, Daubin C, et al. Neurally adjusted ventilatory assist in patients recovering spontaneous breathing after acute respiratory distress syndrome: physiological evaluation. Crit Care Med 2010;38(9):1830-1837. doi:10.1097/ CCM.0b013e3181eb3c51.

[10] Tiffany M, Dixon C, Bozeman R, Patten W. Neurally adjusted ventilatory assist ventilation mode applied in congenital diaphragmatic hernia. Critical Care Medicine 2013;41(12):A297. doi:10.1097/01. ccm.0000440404.58412.fa.

[11] Bengtsson JA, Edberg KE. Neurally adjusted ventilatory assist in children: an observational study. Pediatr Crit Care Med 2010;11(2):253257. doi:10.1097/PCC.0b013e3181b0655e.

[12] Zhu LM, Shi ZY, Ji G, Xu ZM, Zheng JH, Zhang HB, et al. Application of neurally adjusted ventilatory assist in infants who underwent cardiac surgery for congenital heart disease. Zhongguo Dang Dai Er Ke Za Zhi 2009;11(6):433-436.

[13] Firestone KS, Fisher S, Reddy S, White DB, Stein HM. Effect of changing NAVA levels on peak inspiratory pressures and electrical activity of the diaphragm in premature neonates. J Perinatol 2015;35(8):612616. doi:10.1038/jp.2015.14.

[14] Longhini F, Scarlino S, Gallina MR, Monzani A, De Franco S, Grassino $\mathrm{EC}$, et al. Comparison of neurally adjusted ventilator assist in infants before and after extubation. Minerva Pediatr 2015. 